\title{
DNA Mimic Proteins: Functions, Structures, and Bioinformatic Analysis
}

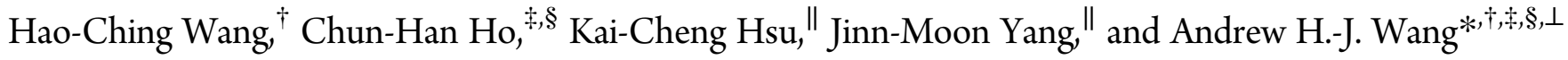 \\ ${ }^{\dagger}$ Graduate Institute of Translational Medicine, College of Medical Science and Technology, Taipei Medical University, Taipei 110, \\ Taiwan \\ ${ }^{\ddagger}$ Institute of Biological Chemistry, Academia Sinica, Taipei 115, Taiwan \\ ${ }^{\S}$ Institute of Biochemical Sciences, National Taiwan University, Taipei 106, Taiwan \\ "Institute of Bioinformatics and Systems Biology and Department of Biological Science and Technology, National Chiao Tung \\ University, Hsinchu 30050, Taiwan \\ ${ }^{\perp}$ Core Facilities for Protein Structural Analysis, Academia Sinica, Taipei 115, Taiwan
}

ABSTRACT: DNA mimic proteins have DNA-like negative surface charge distributions, and they function by occupying the DNA binding sites of DNA binding proteins to prevent these sites from being accessed by DNA. DNA mimic proteins control the activities of a variety of DNA binding proteins and are involved in a wide range of cellular mechanisms such as chromatin assembly, DNA repair, transcription regulation, and gene recombination. However, the sequences and structures of DNA mimic proteins are diverse, making them difficult to predict by bioinformatic search. To date, only a few DNA mimic proteins have been reported. These DNA mimics were

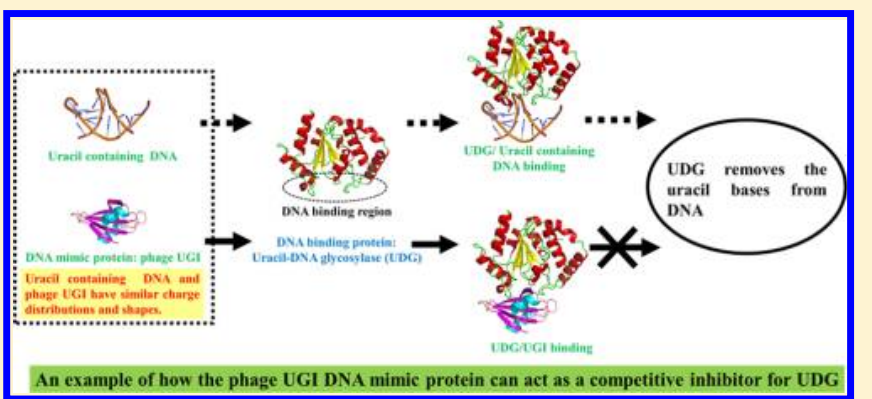
not found by searching for functional motifs in their sequences but were revealed only by structural analysis of their charge distribution. This review highlights the biological roles and structures of 16 reported DNA mimic proteins. We also discuss approaches that might be used to discover new DNA mimic proteins.

$\mathrm{T}$ here are many examples of mimicry in the macroscopic world of plants and animals, but the concept of mimicry also extends to molecular levels. The most well-known examples of mimicry in the cell are enzyme inhibitors. These inhibitors possess chemical properties remarkably similar to those of the normal substrate of their target enzymes, such that competitive inhibition occurs when the "fake" substrate competes with the normal substrate for the active site on the enzyme. This kind of inhibitor includes the sugar-mimicking glycosidase inhibitors and peptide-mimicking protease inhibitors. ${ }^{1,2}$ Compared to other ways of altering enzyme activity, e.g., protein conformational change and post-translational modification, mimicking strategies are relatively simple and direct.

Research in the past decade has revealed several examples of proteins that imitate the substrates of DNA binding proteins. DNA mimic proteins directly occupy the DNA binding sites of these proteins to control their DNA binding activity. ${ }^{3,4}$ DNA mimic proteins have been found in the cells of prokaryotes and eukaryotes as well as in bacteriophages and in a eukaryotic virus. $^{3,5}$ They are involved in many DNA regulation mechanisms, such as the restriction-modification system, ${ }^{6,7}$ the DNA repair activity of uracil-DNA glycosylase, ${ }^{8-13}$ transcriptional control, ${ }^{14-16}$ DNA packaging, ${ }^{5,17-19}$ protection of gyrase from antibiotics, ${ }^{20}$ P53 activity control, ${ }^{21}$ and recombination. $^{22,23}$ In this review, we look at 16 possible
DNA mimic proteins for which annotated functions and protein structures have so far been described (Table 1 and Figure 1).

\section{CHARACTERISTICS OF DNA MIMIC PROTEINS}

For effective mimicry, DNA mimic proteins must have some characteristics that resemble those of a DNA molecule. B-DNA has two regular, helical strands of negative charge distribution, each formed by phosphate groups. Although amino acids, which are the building blocks of proteins, have properties very different from those of the nucleic acid building blocks of DNA, DNA mimic proteins are able to mimic this specific charge distribution with the two negatively charged amino acids: aspartic acid (Asp or D) and glutamic acid (Glu or E). While the known DNA mimic proteins have different structures and their shapes are also very different (see Figure 1), the surface of all of these proteins has distinct negative charge distributions. However, the single-strand mimics DinI and P53 (residues 3360 ) have only a single row, ${ }^{21,22}$ while Gam, which can function as a double-strand or single-strand DNA mimic, has both. ${ }^{23}$ These specialized surface charges can be found on the entire

Received: March 3, 2014

Revised: April 24, 2014

Published: April 25, 2014 
Table 1. Sixteen Previously Identified DNA Mimic Proteins ${ }^{a}$

\begin{tabular}{|c|c|c|c|c|c|c|}
\hline DNA mimic protein & $\begin{array}{l}\text { targeted DNA binding } \\
\text { protein }\end{array}$ & targeted DNA function & $\begin{array}{l}\text { PDB } \\
\text { entry }\end{array}$ & $\begin{array}{l}\text { year of release of } \\
\text { PDB data }\end{array}$ & $\begin{array}{l}\mathrm{MW} \\
(\mathrm{kDa})^{b}\end{array}$ & $\mathrm{pI}^{b}$ \\
\hline \multicolumn{7}{|c|}{ Viruses } \\
\hline UGI (Bacillus phage PBS2) & uracil-DNA glycosylase & DNA repair & 1UGI & 1999 & 9.4 & 4.1 \\
\hline Ocr (enterobacteria phage T7) & type I restriction enzymes & restriction & 1S7Z & 2004 & 13.7 & 3.9 \\
\hline Gam (bacteriophage $\lambda$ ) & RecBCD & recombination & $2 \mathrm{UUZ}$ & 2007 & 11.7 & 5.0 \\
\hline ICP11 (white spot syndrome virus) & histone proteins & nucleosome assembly & 2ZUG & 2008 & 9.2 & 4.2 \\
\hline p56 (Bacillus phage $\phi 29)$ & uracil-DNA glycosylase & DNA repair & 2LE2 & 2011 & 6.5 & 4.2 \\
\hline \multicolumn{7}{|c|}{ Bacteria } \\
\hline DinI (Escherichia coli BL21) & RecA & recombination & $1 \mathrm{GHH}$ & 2001 & 9.0 & 4.7 \\
\hline HI1450 (Haemophilus influenzae PittGG) & $\begin{array}{l}\text { nucleoid-associated protein } \\
\text { HU }\end{array}$ & DNA packaging & $1 \mathrm{NNV}$ & 2004 & 12.6 & 4.0 \\
\hline Mfpa (Mycobacterium tuberculosis) & DNA gyrase & topology & 2BM4 & 2005 & 20.3 & 5.5 \\
\hline NuiA (Nostoc sp.) & nonspecific nuclease & $\begin{array}{l}\text { phosphodiester bond } \\
\text { digestion }\end{array}$ & $2 \mathrm{O} 3 \mathrm{~B}$ & 2006 & 15.1 & 4.4 \\
\hline ArdA (Enterococcus faecalis) & $\begin{array}{l}\text { type I and II restriction } \\
\text { enzymes }\end{array}$ & restriction & $2 W 82$ & 2009 & 19.1 & 3.9 \\
\hline CarS (Myxococcus xanthus) & $\begin{array}{l}\text { CarA transcription } \\
\text { repressor }\end{array}$ & transcription & $2 \mathrm{KSS}$ & 2010 & 9.3 & 4.1 \\
\hline DMP19 (Neisseria meningitidis) & $\begin{array}{l}\text { NHTF transcription } \\
\text { repressor }\end{array}$ & transcription & $3 \mathrm{VJZ}$ & 2012 & 18.5 & 4.4 \\
\hline DMP12 (N. meningitidis) & $\begin{array}{l}\text { nucleoid-associated protein } \\
\text { HU }\end{array}$ & DNA packaging & $3 \mathrm{~W} 1 \mathrm{O}$ & 2013 & 13.9 & 4.6 \\
\hline SAUGI (Staphylococcus aureus) & uracil-DNA glycosylase & DNA repair & $3 W D G$ & 2014 & 13.3 & 4.5 \\
\hline \multicolumn{7}{|c|}{ Eukaryotic Animals } \\
\hline TAFII230 (residues 11-77) (Drosophila) & RNA polymerase & transcription & 1TBA & 1999 & $7.0^{c}$ & $4.0^{c}$ \\
\hline $\begin{array}{l}\text { P53 transactivation domain (residues 33-60) } \\
\text { (Homo sapiens) }\end{array}$ & replication protein $\mathrm{A}$ & single-strand binding & $2 \mathrm{~B} 3 \mathrm{G}$ & 2005 & $3.1^{c}$ & $3.2^{c}$ \\
\hline
\end{tabular}

${ }^{a}$ Functional DNA mimicry has been experimentally demonstrated for all of the DNA mimic proteins listed here. ${ }^{b}$ The theoretical molecular weights (MW) and isoelectric points (pI) are calculated from the amino acid sequences of DNA mimic proteins using ProtParam (http://web.expasy.org/ protparam/). ${ }^{c} \mathrm{MW}$ and $\mathrm{pI}$ are based on the DNA mimic regions of TAFII230 (residues 11-77) and P53 (residues 33-60).

exposed surface of the white spot syndrome virus ICP11 and Haemophilus influenzae HI1450 proteins, ${ }^{5,17}$ on a one-dimensional surface of proteins such as Neisseria DMP19 and bacteriophage PBS2 UGI, ${ }^{8,16}$ or on just a special region of proteins such as eukaryotic TAFII230 (residues 11-77) and P53 (residues 33-60). ${ }^{14,21}$ Figure $2 \mathrm{~A}$ compares the negative charge distribution of two of these different types of DNA mimic proteins (ICP11 and DMP19) with B-form doublestranded DNA at the same scale. The surface charges of DNA mimic proteins and their targets are usually complementary to each other (Figure 2B), suggesting that charge-charge interaction plays an important role in the DNA mimic's ability to bind to its targets. Two single-strand DNA mimic proteins, Gam and $P 53,{ }^{21,23}$ use an additional hydrophobic interaction, whereby aromatic amino acids mimic unpaired bases on the DNA. Because of these characteristics, DNA mimic proteins usually have high binding affinities for their respective targets (Table 2).

It should also be noted that although DNA mimic proteins have DNA-like charge distributions and shapes, they do not exhibit DNA-like structural flexibility. For example, when the three well-studied uracil DNA-glycosylase inhibitors (UGI, SAUGI, and p56) form complexes with their corresponding uracil DNA-glycosylases (UDGs), only very slight conformational changes are seen. ${ }^{8,11,13}$ A somewhat larger change occurs in the NuiA-NucA complex: although binding to NuiA causes only a few minor side chain rearrangements on NucA, on the DNA mimic itself, $\alpha$-helix 4 and several loops are affected. However, the conformations of bound versus unbound NuiA are still close (root-mean-square deviation fit of $3.1 \AA$ ). ${ }^{27}$

\section{VIRAL DNA MIMIC PROTEINS VERSUS HOST DEFENSE MECHANISMS}

In this section, we look at five reported viral DNA mimic proteins, all of which use different means to play an important role against the defense systems of the host.

Ocr. Ocr (overcome classical restriction) is a DNA mimic protein from bacteriophage $\mathrm{T} 7$ that inhibits the activity of the type I R/M system (restriction and modification). ${ }^{25}$ In bacteria, the $\mathrm{R} / \mathrm{M}$ system acts as a defense mechanism against phage infection and other types of DNA invasion. This system consists of a methyltransferase that methylates a specific site on "friendly" DNA to prevent cleavage by a restriction endonuclease. ${ }^{28}$ In this way, host DNA is protected by the sequence-specific base modifications introduced by the DNA methyltransferase (MTase), whereas invading DNA (e.g., a bacteriophage genome) that lacks the appropriate base modifications will be cleaved by the restriction endonuclease. To protect its genomic DNA, a bacteriophage must therefore use some methods to counter the $\mathrm{R} / \mathrm{M}$ system. Bacteriophage T7 Ocr is a small, acidic protein ( $13.7 \mathrm{kDa}$ with pI 3.9) encoded by gene 0.3 , which inhibits all type I R/M enzymatic DNA processing, i.e., both the DNA modification and the DNA cleavage steps. ${ }^{25}$ The crystal structure of Ocr shows that the size of each Ocr monomer is equivalent to $\sim 12$ bp of doublestranded DNA, while the surface charge distribution is a very good match to that of the DNA phosphate group backbone. ${ }^{6}$ The Ocr homodimer forms a croissant shape that mimics the $33.6^{\circ}$ bent B-form DNA, and this allows it to be captured by the type I R/M enzyme with a strong binding affinity in the picomolar range. ${ }^{25,26}$ This model was confirmed for the 


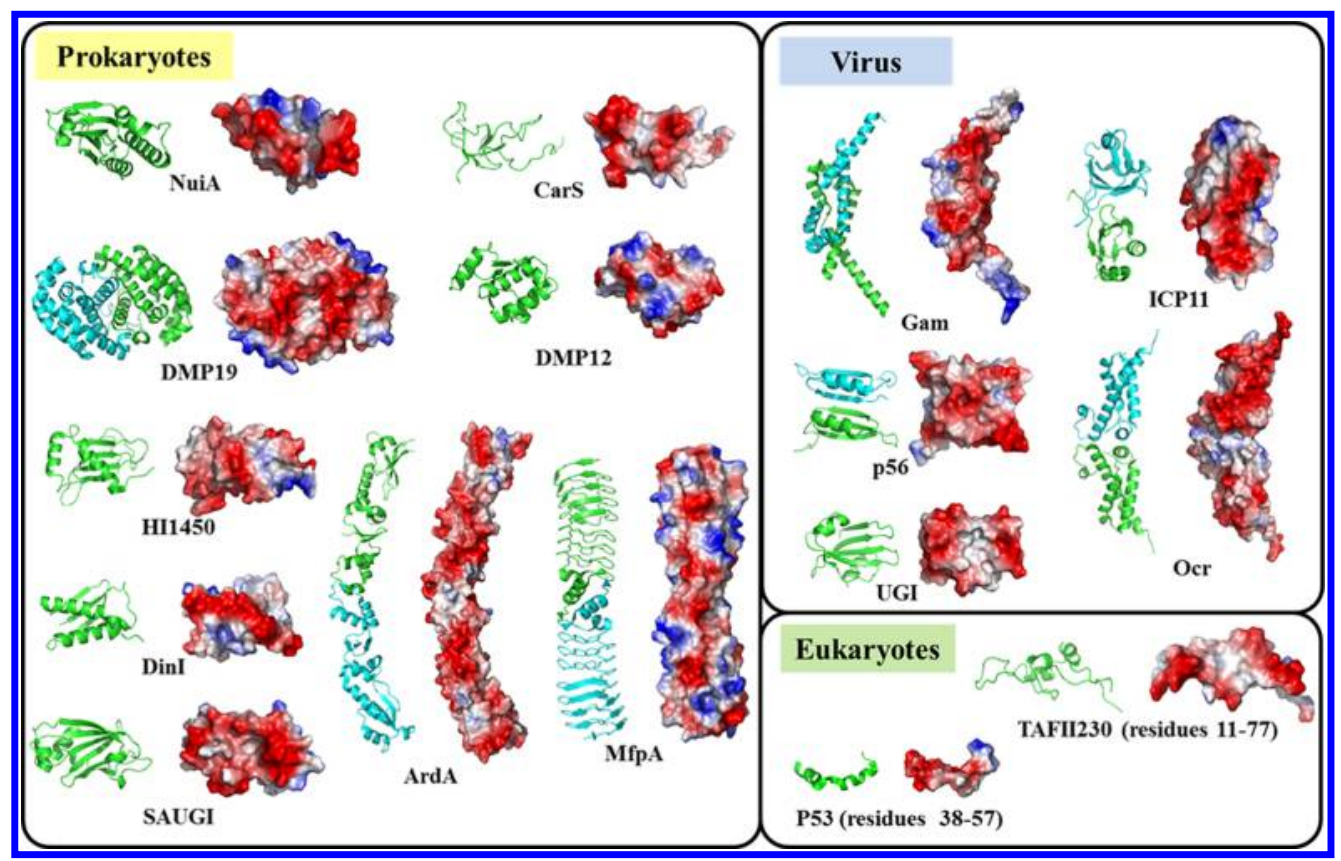

Figure 1. Tertiary structures (ribbon diagrams) and surface charge distributions of 12 published DNA mimic proteins. All of these proteins have double rows of negative charges except for DinI and P53 (single row) and Gam (double rows and an additional single row). In bacteria and viruses, DNA mimicry is performed by small, acidic proteins, while in the two known eukaryotic DNA mimics, the mimicry is performed by a motif or domain within the protein. The shapes of DNA mimic proteins can be very different. For example, Mycobacterium tuberculosis MfpA uses a righthanded $\beta$-helix to form a rodlike shape, while bacteriophage Ocr has a croissantlike shape that mimics bent double-stranded DNA. Meanwhile, Escherichia coli DinI, Haemophilus influenzae HI1450, and white spot syndrome virus ICP11 all have a globular protein surface. The secondary structures seen in these DNA mimic proteins include all known secondary structure types, i.e., $\alpha$-helices, $\beta$-strands, and peptide loops, and structural homology searches via the DALI Web site reveal no consistent features. Because the amino acid sequences are also thought to be unique (no primary structure similarity has yet been reported for the published DNA mimic proteins), it is hard to identify DNA mimic proteins by using bioinformatic searches.

M.EcoKI type I R/M enzyme-Ocr binding complex by image reconstruction from electron micrographs. ${ }^{29}$

UGI and p56. UGI from Bacillus phage PBS2 is another example of a phage DNA mimic protein that acts as a uracilDNA glycosylase inhibitor. ${ }^{30}$ Bacillus phage PBS2 contains a double-stranded DNA genome that is unusual in that it incorporates uracil nucleotides instead of thymine nucleotides. This special genetic code of PBS2 prevents the phage genome from being attacked by the R/M system. However, bacteria also contain a DNA repair enzyme called uracil-DNA glycosylase (UDG) that acts to remove uracil bases from damaged DNA in a repair process that has two steps. (1) UDG docks on the DNA uracil site, and (2) it flips the uracil base out of the stacked double-stranded DNA. PBS2 therefore uses UGI to counter this repair mechanism, which would otherwise lead to the inactivation of the PBS2 genome and prevent viral replication. ${ }^{8,30}$ Functionally, UGI blocks UDG by irreversibly forming a UDG-UGI complex. It is interesting to note that UGI mimics not only the shape and charge distribution of DNA but also the specific "flipped" state that uracil-DNA adopts after being bound by UDG. This additional level of mimicry allows UGI to block UDGs from a wide range of sources, from prokaryotic cells to eukaryotic cells and viruses, while still being highly specific. Thus, UGI targets the UDGs from B. subtilis, E. coli, Micrococcus luteus, Saccharomyces cerevisae, rat liver, herpes simplex virus (HSV), and humans, but it does not target other common DNA-metabolizing enzymes or even other DNA glycosylases. ${ }^{8}$

Recently, a novel uracil-DNA glycosylase inhibitor, p56, was found in Bacillus phage $\phi 29 .{ }^{9,10}$ Although the structures of dimeric p56 and monomeric UGI are quite different, dimeric p56 also forms a stable complex with Bacillus or HSV UDG in a 2:1 molar stoichiometry. ${ }^{11,12}$ Unlike phage PBS2, the genomic DNA of phage $\phi 29$ does not contain uracil nucleotides, but Serrano-Heras hypothesized that p56 might knock down this UDG activity to prevent uracil nucleotides from appearing in the replicative intermediates during phage replication. ${ }^{9}$

Gam. Lambda phage Gam is a DNA mimic protein that inhibits all the known enzymatic activities of the bacterical protein complex RecBCD, which is an exonuclease and helicase that also acts as a defense mechanism against bacteriophage infection. ${ }^{31}$ RecBCD works by digesting the linear viral DNA that is present during replication of the virus or results from the action of restriction enzymes, and Murphy showed that Gam acted to prevent RecBCD from binding to DNA. ${ }^{32}$ The crystal structure of Gam suggests it may mimic both single-stranded and double-stranded DNA. ${ }^{23}$ The N-terminal $\alpha$-helix 1 of Gam might act as a single-strand DNA mimic that enters either the $3^{\prime}$ channel on RecB or the $5^{\prime}$ channel of RecC/RecD, while the shape, dimensions, and negative charge of the Gam dimerization domain led Court et al. to further suggest that, like Ocr, Gam might use this domain to mimic the charge and shape of the double-stranded DNA. ${ }^{23}$ However, the actual structure of the Gam-RecBCD complex still needs to be clarified experimentally to confirm this hypothesis.

ICP11. White spot syndrome virus (WSSV), a doublestranded DNA virus that causes a lethal disease in shrimp, expresses the nonstructural protein ICP11 at the late stage of viral replication. ${ }^{33}$ The role of ICP11 as a DNA mimic was suggested by structural analysis, which showed that the ICP11 


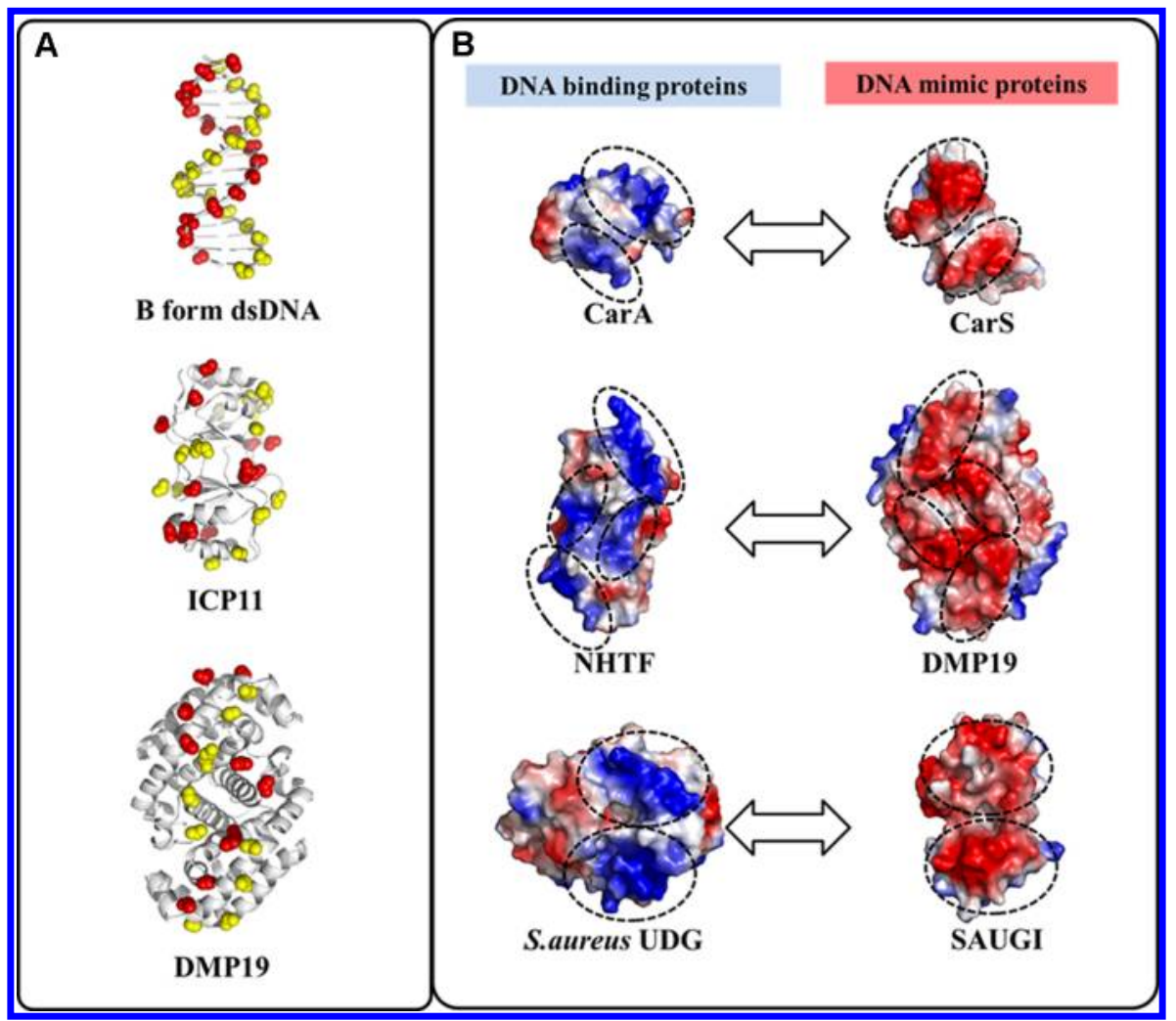

Figure 2. Specialized configuration of the surface charges on the surface of DNA mimic proteins. (A) These space-filling models show how the phosphate groups on the DNA backbone of B-form double-stranded DNA are mimicked by the negatively charged carboxyl groups of ICP11 and DMP19. The two negatively charged rows in each molecule are colored red and yellow, respectively. (B) Matching surface configuration of DNA mimic proteins and their target DNA binding proteins. Surface charges for the first two pairs were obtained from the individual proteins of the proposed binding models, while the $S$. aureus UDG/SAUGI surface charges were obtained from the actual complex.

Table 2. Binding Affinities of DNA Mimic Proteins and Their Respective Targets

\begin{tabular}{|c|c|c|c|}
\hline DNA mimic protein & targeted DNA binding protein & binding affinity ( $K_{\mathrm{D}}$, equilibrium constant) & refs \\
\hline \multicolumn{4}{|c|}{ Binding Affinities Determined by Surface Plasmon Resonance (BIAcore) } \\
\hline UGI (Bacillus phage PBS2) & S. aureus uracil-DNA glycosylase & $0.69 \mathrm{nM}$ & 13 \\
\hline DinI (E. coli BL21) & E. coli RecA & $0.86 \mu \mathrm{M}$ & 24 \\
\hline Mfpa (M. tuberculosis) & E. coli DNA gyrase & $0.46 \mu \mathrm{M}$ & 20 \\
\hline SAUGI (S. aureus) & S. aureus uracil-DNA glycosylase & $1.20 \mathrm{nM}$ & 13 \\
\hline \multicolumn{4}{|c|}{ Binding Affinities Determined by Isothermal Titration Calorimetry (ITC) } \\
\hline Ocr (enterobacteria phage $\mathrm{T} 7$ ) & E. coli M.EcoKI & $<100 \mathrm{pM}^{a}$ & 25,26 \\
\hline p56 (Bacillus phage $\phi 29)$ & Bacillus subtilis uracil-DNA glycosylase & $16.00 \mathrm{nM}$ & 10 \\
\hline HI1450 (H. influenzae PittGG) & H. influenzae $\mathrm{HU} \alpha$ & $3.00 \mu \mathrm{M}$ & 18 \\
\hline DMP12 (N. meningitidis) & N. meningitidis $\mathrm{HU}$ & $2.82 \mu \mathrm{M}$ & 19 \\
\hline CarS (M. xanthus) & M. xanthus CarA N-terminal domain (residues 1-79) & $12.00 \mathrm{nM}$ & 15 \\
\hline
\end{tabular}

dimer has two rows of negatively charged spots that approximately match, in scale and configuration, the arrangement of the phosphate groups in the two opposing strands of DNA (Figure 2). ${ }^{5}$ Functionally, ICP11 prevents DNA from binding to histone proteins $\mathrm{H} 2 \mathrm{~A}, \mathrm{H} 2 \mathrm{~B}, \mathrm{H} 3$, and $\mathrm{H} 2 \mathrm{~A}$.x. In WSSV-infected shrimp hemocytes and ICP11-expressing HeLa cells, the predominantly cytoplasmic colocalization of ICP11 with histone $\mathrm{H} 3$ and histone H2A.x suggests that ICP11 prevents these histones from translocating into the nucleus where they would normally participate in nucleosome assembly or fulfill some other histone function. Although the exact purpose of ICP11's DNA mimicry is not yet clear, the high expression level of this protein suggests that it is likely to be important in WSSV replication. It may be that by disrupting the host's nucleosomes, ICP11 affects the epigenetic control of gene expression. If so, it might also have the presumably unwanted consequence of inducing cell death. ${ }^{5}$ Histone proteins and their derived fragments also have antimicrobial activity in vertebrates and invertebrates, and many studies have focused on the defensive role of $\mathrm{H} 2 \mathrm{~A}$ and $\mathrm{H} 2 \mathrm{~B}$. For example, in the Pacific white shrimp Litopenaeus vannamei, enzymatic cleavage of histone $\mathrm{H} 2 \mathrm{~A}$ produces active peptides that can completely inhibit the growth of Gram-positive bacteria. ${ }^{34}$ Thus, it is also possible that ICP11's ability to bind to histone proteins might be a direct attempt to interfere with the host's defenses. 
BACTERIAL DNA MIMIC PROTEINS ACT AS INTERNAL CONTROL FACTORS

Approximately half of the reported DNA mimic proteins were found in bacteria. DNA mimic proteins such as $\operatorname{ArdA}^{7}$ HI1450, ${ }^{17,18}$ and $\mathrm{MfpA}^{20}$ are conserved in many different species, suggesting that they are important to prokaryotes. Unlike the viral DNA mimic proteins, the bacterial DNA mimics act not only as inhibitors but also as regulators. They are involved in many internal response controls.

Dinl. E. coli DinI is a bacterial protein that inhibits the activity of RecA and limits the response of the SOS mutagenesis system to DNA damage. ${ }^{24,35}$ The solution structure of DinI provided some insight into how it might achieve RecA inactivation by acting as a single-strand DNA mimic protein. ${ }^{22}$ The C-terminal $\alpha$-helix of DinI contains six negatively charged Asp and Glu R.EcoKI residues, and these amino acids are arranged in an extended, negatively charged ridge. Because this ridge has an electrostatic character similar to that of the phosphate backbone of single-stranded DNA, DinI may use this $\alpha$-helical region to compete with single-stranded DNA in binding to the L2 loop of RecA. In the model proposed by Ramirez et al., ${ }^{22}$ DinI displaces the single-stranded DNA from the active RecA nucleoprotein filament, inhibits the strand exchange reaction, and thus destabilizes filament formation. However, the actual mechanism of the RecA-DinI interaction is still in dispute. More recent reports have shown that the interactions of DinI with the RecA filament are concentration-dependent and may in fact result in either stabilization or depolymerization of the filament. ${ }^{36,37}$ More studies will be needed to fully elucidate the functional effects of DinI on RecA filament formation.

HI1450 and DMP12. Homologues of the H. influenzae HI1450 protein are widely distributed and can be found in many different species, including Salmonella typhimurim, Yersinia pestis, E. coli, and Vibrio vulnificus. ${ }^{17,18} \mathrm{HI} 1450$ is weakly structurally homologous to UGI, while its doublestranded DNA-like surface charge distribution led Parsons to propose that it might act as a B-form DNA mimic protein. ${ }^{17}$ Functionally, HI1450 prevents DNA from binding to the bacterial nucleoid-associated protein $\mathrm{HU}_{-} \alpha$, and it may therefore have a role in controlling the chromosome-like structure in prokaryotes. ${ }^{18}$ However, the full biological meaning of the HI1450-HU- $\alpha$ interaction has yet to be experimentally determined.

DMP12 is another DNA mimic protein that binds to HU, and it is found in Neisseria spp. ${ }^{19}$ The DMP12 monomer has a specialized shape and charge distribution that resembles those of HU-bound DNA and is complementary to the HU protein dimer. In a competitive electrophoresis mobility shift assay (EMSA), DMP12 only partially disrupted the formation of HU-plasmid DNA complexes. The binding affinities determined by isothermal titration calorimetry also showed that the affinity of Neisseria HU for DMP12 is 4 times lower than it is for dsDNA. These findings suggest that although DMP12 and DNA share the same binding sites on the HU protein, DMP12 is more likely to act as a regulator than a competitive inhibitor. Because the DNA binding $\beta$-ribbon arms of bacterial $\mathrm{HU}$ protein are usually disordered and unstable, DMP12 may act to maintain the stability of the disordered regions of the unbound HU protein. This hypothesis was investigated using a limited trypsin digestion assay that showed Neisseria HU protein is sensitive to trypsin in the absence of DMP12 or dsDNA, while
DMP12 and dsDNA both reduced the rate of HU protein degradation.

ArdA. Bacteria also express antirestriction proteins. Although these are functionally similar to the Ocr protein of phage T7, the roles played by these bacterial mimics are somewhat different. ArdA protein is very commonly found in conjugative plasmids and transposons in a wide array of prokaryotes. $^{38}$ Compared to Ocr, ArdA is a more effective inhibitor of the activity of the M.EcoKI type IA restriction enzyme in the type I R/M system, and this inhibition allows the horizontal transfer of mobile genetic DNA elements (e.g., plasmid DNA) between different bacterial cells. ${ }^{39}$ It was recently shown that the rodlike structure of dimeric ArdA mimics $\sim 42$ bp of bent B-form DNA, which is close in length to the normal DNA substrate of the M.EcoKI type IA restriction enzyme. ${ }^{7}$ The carboxyl groups on the surface of the ArdA dimer also match the negatively charged phosphate groups of the DNA that is bound to the type I MTase core. Interestingly, the mutation that disrupts the dimeric structure of ArdA will affect ArdA's antimodification activity, but not its antirestriction activity. ${ }^{40}$ This suggests that, in addition to the dimeric binding of ArdA to the core MTase, the ArdA monomer might also bind independently to the restriction subunit of the type I restriction enzyme. The ability of both Ocr and ArdA proteins to inhibit the type $\mathrm{I} R / \mathrm{M}$ system despite their different structures and binding affinities is a striking reminder of the diversity found among DNA mimic proteins even when they fulfill similar functional roles.

MfpA. Fluoroquinolones are powerful antibiotics that prevent bacterial DNA from unwinding and duplicating by inhibiting the activity of DNA gyrase and topoisomerase IV. However, resistance to fluoroquinolones is a clinical problem in the treatment of $M$. tuberculosis. The MfpA gene/protein was first identified from fluoroquinolone-resistant strains of $M$. tuberculosis. ${ }^{41}$ Functionally, MfpA protein binds to the DNA binding site of gyrase and protects it from fluoroquinolones. ${ }^{42}$ Analysis of the crystal structure of MfpA revealed that the dimeric form presents as a right-handed quadrilateral $\beta$-helix and rodlike structure. ${ }^{20}$ Although the $\mathrm{pI}$ of MfpA (5.5) is higher than those of other DNA mimic proteins, the pattern of distribution of the acidic amino acids on the protein surface still creates enough negatively charged spots to mimic a $30 \mathrm{bp} \mathrm{B-}$ form DNA. ${ }^{20} \mathrm{MfpA}$ is the first antibiotic-resistance protein that protects the antibiotic's target directly by binding to it, rather than by degrading the drug. Since this discovery, quinoloneresistance proteins QnrB1 [Protein Data Bank (PDB) entry 2XTW], AlbG (PDB entry 2XT2), and EfsQnR (PDB entry 2W7Z) have also been shown to have a similar structure, suggesting that they might use the same strategy against this related antibiotic family. ${ }^{43-45}$ Interestingly, quinolone-related antibiotics such as the fluoroquinolones are a relatively recent invention, which raises the question of what the original function of MfpA might have been. It should be interesting to investigate other possible biological functions of $\mathrm{MfpA}$ and its homologues.

NuiA. Nuclease A (NucA) is a nonspecific endonuclease from Anabaena sp. that digests single- and double-stranded DNA and RNA in the presence of divalent metal ions. ${ }^{46}$ Nonspecific nucleases are involved in a broad range of functions, including programmed cell death, defense, DNA replication, recombination, and repair, and in general, their activities must be carefully controlled to ensure that they do not become toxic to the cell. ${ }^{27}$ NuiA is a specific inhibitor of NucA 
that uses the negatively charged amino acids located at the edge of its central $\beta$-sheet to mimic the phosphate backbone of NucA's DNA substrate. Additionally, the Thr135 residue on the C-terminus of NuiA is directly coordinated with the catalytic $\mathrm{Mg}^{2+}$ of the NucA active site, while the Glu24 residue of NuiA also extends into the active site, mimicking the charge of a scissile phosphate. NucA and NuiA thus form a tight 1:1 complex with picomolar affinity. ${ }^{27}$

CarS. In M. xanthus, CarA-CarS repressor-antirepressor interaction is involved in the transcriptional control of the carB operon that responds to the carotenogenesis induced by blue light. ${ }^{47,48}$ In the dark, RNA polymerase cannot access the promoter on the carB operon because it is blocked by CarA, which binds cooperatively to a bipartite operator, one part of which overlaps with the -35 promoter region. ${ }^{49}$ This inhibition by CarA is neutralized by the CarS antirepressor that is expressed in blue light. ${ }^{47}$ CarS recognizes the MerR-type, winged-helix DNA-binding domain on the N-terminus of CarA, and structural analysis showed that CarS contains a polar solvent-exposed pocket that is filled with negatively charged residues. ${ }^{15}$ This pocket mimics (with some slight distortion) the major groove of the operator DNA, and it binds to CarA recognition helix $\alpha 2$ with high affinity $\left(K_{\mathrm{D}} \sim 10 \mathrm{nM}\right)$. This allows CarS to compete effectively against the operator DNA in trapping the CarA DNA binding domain. ${ }^{15}$

DMP19. Neisseria DMP19 is a conserved gene/protein that is found in the major hypervirulent lineages and serogroups of many Neisseria species that cause human disease. ${ }^{50,51}$ Surface charge analysis revealed a double-stranded DNA-like negative charge distribution on one of the dimensional electrostatic surfaces of the DMP19 dimer. ${ }^{16}$ Functional studies showed that DMP19 prevented a Neisseria transcriptional repressor (NHTF) from binding to its DNA target, which is a specific sequence in its own promoter region. The complementary surface charges of DMP19 and NHTF strongly suggest that a charge-charge interaction mediates the binding of these two proteins. Because NHTF is an autorepressor that downregulates its own expression as well as the expression of downstream genes, there are likely to be critical situations in which more control is needed. DMP19 is thought to play a role in the further control of NHTF's autofeedback mechanism: by temporarily interfering with the binding of NHTF to its own promoter region, DMP19 also restores the expression of the downstream genes. NHTF belongs to the XRE transcription factor family, and it will be interesting to determine whether DMP19 can interact with other members of the same family.

SAUGI. The most recently reported example of a DNA mimic protein is Staphylococcus SAUGI. SAUGI is the third uracil-DNA glycosylase inhibitor to be identified, and the first to be found in a non-phage species. ${ }^{13}$ Like the two other uracil DNA-glycosylase inhibitors (UGI and p56), ${ }^{8-12}$ SAUGI acts by binding tightly to Staphylococcus UDG or human UDG with a nanomolar affinity. Although the amino acid sequences and structures of SAUGI, UGI, and p56 are different, all three of these uracil DNA-glycosylase inhibitors share a conserved UDG binding strategy that involves targeting their respective UDG's protruding residue (i.e., SAUDG Leu184, E. coli UDG Leu191, and B. subtilis UDG Phe191) with a hydrophobic pocket. The three uracil-DNA glycosylase inhibitors are also similar in that when they bind to their respective targets, they induce only very minor conformational changes in the UDGs. However, unlike UGI and p56, which help their respective phages to replicate by inhibiting host UDG activity, it is not clear why
Staphylococcus would produce a protein that inhibits its own DNA repair system. Perhaps, like bacteria ArdA, which inhibits the bacteria's own restriction enzymes to allow the horizontal transfer of mobile genetic DNA elements, ${ }^{38,39}$ SAUGI also acts to regulate UDG activity for some as yet unknown purpose. For instance, because it has been shown that the replication of DNA viruses can be blocked by increasing the amount of dUTP in the viral genome, ${ }^{52,53}$ SAUGI's inhibition of UDG might serve an anti-phage function by increasing the amount of uracil on the phage genome. ${ }^{13}$

\section{EUKARYOTIC P53 AND TAFII230 PROTEINS USE MOTIFS TO ACHIEVE DNA MIMICRY}

Most DNA mimic proteins from bacteria and viruses use the entire protein to produce a DNA-like charge distribution on the protein surface. By contrast, the two eukaryotic examples that are presently known, P53 and TAFII230, use only a part of the protein to mimic DNA. Both of these proteins are involved in transcription control.

P53. The first of these eukaryotic proteins, P53, is a wellknown tumor suppressor. When it is activated, it modulates the transcriptional regulation of genes involved in the cell cycle, DNA repair, and apoptosis. ${ }^{34}$ P53 protein interacts with a number of targets, including DNA as well as other types of proteins. One of P53's targets is replication protein A (RPA), which is the major single-strand DNA binding protein of the eukaryotic nucleus. ${ }^{55}$ The interaction between P53 and RPA suppresses homologous recombination and modulates Werner syndrome helicase activity. ${ }^{56,57}$ Structural analysis of the complex structure formed by the $\mathrm{N}$-terminal acidic transcription-activation domain (P53TAD) and RPA showed how these two proteins interact with each other. ${ }^{21}$ A comparison of the complex formed by RPA and the P53TAD and that formed by RPA and single-stranded DNA showed that the negatively charged and hydrophobic amino acids on $\alpha$-helix 1 of the P53TAD are able to mimic the phosphate backbone and bases of the RPA-bound single-stranded DNA. The P53TAD is in competition with single-stranded DNA to bind to RPA, and increasing the local concentration of single-stranded DNA causes the RPA-P53 complex to dissociate. Because singlestranded DNA is produced as a consequence of DNA damage, P53 may be using its single-stranded DNA mimic region to control the activity of RPA. Recently, P53 was also found to interact with positive cofactor 4 (PC4) via the same P53TAD. ${ }^{58}$ PC4 is a transcriptional coactivator that mediates activatordependent transcription of class II genes through interactions with the basal machinery. ${ }^{59}$ The C-terminal DNA binding domain of PC4 binds tightly to both single-stranded DNA and to melted double-stranded DNA, but the P53TAD competes with this domain only for single-stranded DNA. ${ }^{58}$ Other acidic transcription-activation domains can be found on herpes simplex virion protein 16 (VP16) ${ }^{60}$ and eukaryotic transcriptional regulator Ap- $2 \alpha{ }^{61}$ The similarity of these domains to P53TAD suggests that these proteins might also use the same single-stranded DNA mimic strategy to modulate transcription.

TAFII230. One of the proteins in the eukaryotic TFIID complex also shows DNA mimic activity. The TFIID complex acts as a general transcription factor that is involved in eukaryotic transcription initiation, and it consists of the TATA box-binding protein (TBP) and several TBP-associated factors. The complex plays a central role in both positive and negative transcriptional control. ${ }^{62}$ Drosophila TATA box-binding TAFII (TAFII230) is a $230 \mathrm{kDa}$ protein that prevents TBP from 


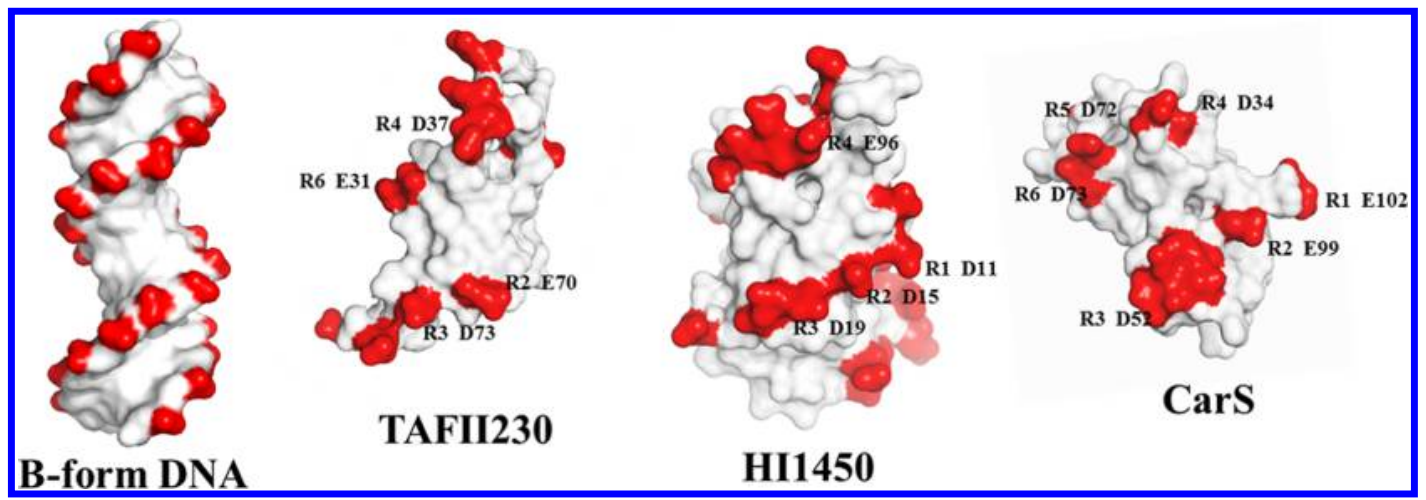

Figure 3. Conserved negative charge distribution and shape of three DNA mimic proteins. The locations of the conserved negatively charged spots match the locations of those of B-form double-stranded DNA on these three individual DNA mimic proteins. The six matching positions are labeled R1-R6.

binding to the TATA box. Structural analysis of the protein complex shows that the N-terminal domain of TAFII230 (residues 11-77) binds to TBP by mimicking the minor groove surface of the TATA box. ${ }^{14}$ Because gene expression is shut down when TBP binds to the TATA box, the TAFII230-TBP interaction is likely to play an important role in the transcriptional control of the genes downstream of the TATA box.

\section{PREDICTING NEW DNA MIMIC PROTEINS BY BIOINFORMATIC ANALYSIS}

Although we have reviewed some of the ways in which how DNA mimics function, the lack of any conserved functional motif or domain on these 16 DNA mimic proteins means that this information is not immediately helpful when searching for new DNA mimic candidates. Additionally, the published DNA mimic proteins do not have a conserved structure, and in fact, they are often classified as proteins that have novel folding because of their lack of homology to any known structure. Conversely, even when these proteins contain the same secondary structures, these structures are arranged in different ways. Thus, for example, as shown in Figure 1 for DMP19, Gam, and Ocr, although each of these proteins consists entirely of $\alpha$-helices, the actual folding of each respective protein is quite different. Although DNA mimic proteins from bacteria and viruses are usually small and acidic, this too is still not specific enough to identify new DNA mimic proteins because many other proteins, such as ferritin, SUMO proteins, and calmodulin, also have these two features. It seems likely that the only shared, universal feature of DNA mimic proteins is the negative charge distribution on their protein surface, and this is hard to predict without knowledge of the three-dimensional structure of the protein. Thus, while a handful of DNA mimic proteins have so far been reported, it is highly possible that the protein database already contains many unrecognized protein structures that mimic DNA. The fact that SAUGI was already identified as a DNA mimic protein is a case in point. ${ }^{13}$

A new approach that may potentially be useful for identifying DNA mimic proteins is to consider the tertiary structure of candidate mimic proteins. A comparison of the 16 reported DNA mimic proteins shows that the negatively charged spots of three of them (TAF230, HI1450, and CarS) have a similar pattern. A superimposition of these four DNA mimic proteins over double-stranded DNA shows how six of the negatively charged amino acids on these three DNA mimic proteins are aligned [R1-R6 (Figure 3)]. The alignment of these proteins seems to suggest that these sites must be conserved for the protein to perform DNA mimic activity. We also note that the shapes of the regions between the negatively charged rows resemble DNA grooves (Figure 3).

\section{CONCLUDING REMARKS}

Although the biological role of the DNA mimic protein OCR was first reported by William Studier more than 30 years ago, ${ }^{63}$ there are still only a handful of known DNA mimics. Even so, DNA mimics have already been shown to have several potentially important applications. (1) The DNA mimic protein Ocr significantly increases transformation efficiencies of unmodified DNA in bacterial strains by inhibiting their $\mathrm{R} / \mathrm{M}$ systems. ${ }^{64}$ (2) While a monomeric mutant of the Ocr is already being used as a novel fusion tag to improve the solubility of recombinant proteins, ${ }^{65}$ Ratner previously reported that Ocr protein binds to a column containing immobilized RNA polymerase, ${ }^{66}$ which suggests that it might be possible to use Ocr as a new affinity tag. (3) The uracil-DNA glycosylase inhibitors UGI and SAUGI and the histone binding protein ICP11 are able to bind to their respective targets in mammalian cells, and this has potential applications in human research. Another example is the fluoroquinolone-resistance protein MfpA, which could become an important target in clinical treatment. It should also be possible to design peptide inhibitors for other disease-related DNA binding proteins such as NF $\kappa$ B by using a DNA mimic strategy.

As more DNA mimics are discovered, it seems likely that the number of potential applications will also increase. Some new candidate DNA mimics are already being reported, but there must be a vast number of other DNA mimic proteins that have not yet been identified and investigated. While the best empirical evidence of actual DNA mimicry is provided by solid cocrystal structural data for the interactions between DNA mimic proteins and their targets, an effective platform of bioinformatic screening would be still extremely useful for finding new DNA mimics. As we have shown here, the critical characteristics of DNA mimic proteins typically include a small size, a low pI, a DNA-like negative charge distribution on the protein's surface, and the conserved positions of the negatively charged amino acids on the surface that matches the DNA groove. Although the research into this family of novel control factors is still in its infancy, all of these characteristics are potentially useful for bioinformatic searches in the Protein Data Bank. 


\section{AUTHOR INFORMATION}

\section{Corresponding Author}

*Institute of Biological Chemistry, Academia Sinica, Taipei 115, Taiwan. E-mail: ahjwang@gate.sinica.edu.tw. Telephone: 8862-2788-1981. Fax: 886-2-2788-2043.

\section{Funding}

This work was supported financially by Academia Sinica, the National Science Council, and Taipei Medical University (Grants 022371, NSC102-2319-B-001-003, NSC102-2311-B038-003, and TMU102-AE1-B12).

\section{Notes}

The authors declare no competing financial interest.

\section{ACKNOWLEDGMENTS}

We thank Prof. Ming-Daw Tasi of Academia Sinica for his helpful criticism.

\section{ABBREVIATIONS}

Ocr, overcome classical restriction; R/M system, restriction and modification system; UGI, phage PBS2 uracil-DNA glycosylase inhibitor; p56, phage $\phi 29$ protein of 56 amino acids; UDG, uracil-DNA glycosylase; HSV, herpes simplex virus; WSSV, white spot syndrome virus; ICP11, intracellular protein of 11 $\mathrm{kDa}$; DinI, DNA damage-inducible protein I; M.EcoKI, methyltransferase subunit of the EcoKI restriction-modification system; R.EcoKI, restriction endonuclease subunit of the EcoKI restriction-modification system; MTase, DNA methyltransferase; NuiA, nuclease A inhibitor; NucA, nuclease A; MfpA, Mycobacterium fluoroquinolone resistance protein A; ArdA, anti-restriction protein type A; DMP19 and DMP12, Neisseria DNA mimic proteins of 19 and $12 \mathrm{kDa}$, respectively; NHTF, Neisseria hypothetical transcriptional factor; SAUGI, $S$. aureus uracil-DNA glycosylase inhibitor; SAUDG, S. aureus uracil-DNA glycosylase; TAFII230, transcription fator II D 230 $\mathrm{kDa}$ subunit.

\section{REFERENCES}

(1) Asano, N. (2009) Sugar-mimicking glycosidase inhibitors: Bioactivity and application. Cell. Mol. Life Sci. 66, 1479-1492.

(2) Abbenante, G., and Fairlie, D. P. (2005) Protease inhibitors in the clinic. Med. Chem. 1, 71-104.

(3) Dryden, D. T. F. (2006) DNA mimicry by proteins and the control of enzymatic activity on DNA. Trends Biotechnol. 4, 378-382.

(4) Putnam, C. D., and Tainer, J. A. (2005) Protein mimicry of DNA and pathway regulation. DNA Repair 4, 1410-1420.

(5) Wang, H. C., Wang, H. C., Ko, T. P., Lee, Y. M., Leu, J. H., Ho, C. H., Huang, W. P., Lo, C. F., and Wang, A. H. J. (2008) White spot syndrome virus protein ICP11: A histone-binding DNA mimic that disrupts nucleosome assembly. Proc. Natl. Acad. Sci. U.S.A. 105, 20758-20763.

(6) Walkinshaw, M. D., Taylor, P., Sturrock, S. S., Atanasiu, C., Berge, T., Henderson, R. M., Edwardson, J. M., and Dryden, D. T. (2002) Structure of Ocr from bacteriophage T7, a protein that mimics B-form DNA. Mol. Cell 9, 187-194.

(7) McMahon, S. A., Roberts, G. A., Johnson, K. A., Cooper, L. P., Liu, H., White, J. H., Carter, L. G., Sanghvi, B., Oke, M., Walkinshaw, M. D., Blakely, G. W., Naismith, J. H., and Dryden, D. T. (2009) Extensive DNA mimicry by the ArdA anti-restriction protein and its role in the spread of antibiotic resistance. Nucleic Acids Res. 37, 48874897.

(8) Putnam, C. D., Shroyer, M. J., Lundquist, A. J., Mol, C. D., Arvai, A. S., Mosbaugh, D. W., and Tainer, J. A. (1999) Protein mimicry of DNA from crystal structures of the uracil-DNA glycosylase inhibitor protein and its complex with Escherichia coli uracil-DNA glycosylase. J. Mol. Biol. 287, 331-346.

(9) Serrano-Heras, G., Ruiz-Masó, J. A., del Solar, G., Espinosa, M., Bravo, A., and Salas, M. (2007) Protein p56 from the Bacillus subtilis phage $\phi 29$ inhibits DNA-binding ability of uracil-DNA glycosylase. Nucleic Acids Res. 35, 5393-5401.

(10) Asensio, J. L., Pérez-Lago, L., Lázaro, J. M., González, C., Serrano-Heras, G., and Salas, M. (2011) Novel dimeric structure of phage $\varphi 29$-encoded protein p56: Insights into uracil-DNA glycosylase inhibition. Nucleic Acids Res. 39, 9779-9788.

(11) Baños-Sanz, J. I., Mojardín, L., Sanz-Aparicio, J., Lázaro, J. M., Villar, L., Serrano-Heras, G., González, B., and Salas, M. (2013) Crystal structure and functional insights into uracil-DNA glycosylase inhibition by phage $\phi 29$ DNA mimic protein p56. Nucleic Acids Res. 41, 6761-6773.

(12) Cole, A. R., Ofer, S., Ryzhenkova, K., Baltulionis, G., Hornyak, P., and Savva, R. (2013) Architecturally diverse proteins converge on an analogous mechanism to inactivate uracil-DNA glycosylase. Nucleic Acids Res. 41, 8760-8775.

(13) Wang, H. C., Hsu, K. C., Yang, J. M., Wu, M. L., Ko, T. P., Lin, S. R., and Wang, A. H. J. (2014) Staphylococcus aureus protein SAUGI acts as a uracil-DNA glycosylase inhibitor. Nucleic Acids Res. 42, 13541364.

(14) Liu, D., Ishima, R., Tong, K. I., Bagby, S., Kokubo, T. Muhandiram, D. R., Kay, L. E., Nakatani, Y., and Ikura, M. (1998) Solution structure of a TBP-TAF(II)230 complex: Protein mimicry of the minor groove surface of the TATA box unwound by TBP. Cell 94, $573-583$.

(15) León, E., Navarro-Avilés, G., Santiveri, C. M., Flores-Flores, C., Rico, M., González, C., Murillo, F. J., Elías-Arnanz, M., Jiménez, M. A., and Padmanabhan, S. (2010) A bacterial antirepressor with SH3 domain topology mimics operator DNA in sequestering the repressor DNA recognition helix. Nucleic Acids Res. 38, 5226-5241.

(16) Wang, H. C., Ko, T. P., Wu, M. L., Ku, S. C., Wu, H. J., and Wang, A. H. J. (2012) Neisseria conserved protein DMP19 is a DNA mimic protein that prevents DNA binding to a hypothetical nitrogenresponse transcription factor. Nucleic Acids Res. 40, 5718-5730.

(17) Parsons, L. M., Yeh, D. C., and Orban, J. (2004) Solution structure of the highly acidic protein HI1450 from Haemophilus influenzae, a putative double-stranded DNA mimic. Proteins 54, 375383.

(18) Parsons, L. M., Liu, F., and Orban, J. (2005) HU- $\alpha$ binds to the putative double-stranded DNA mimic HI1450 from Haemophilus influenzae. Protein Sci. 14, 1684-1687.

(19) Wang, H. C., Wu, M. L., Ko, T. P., and Wang, A. H. J. (2013) Neisseria conserved hypothetical protein DMP12 is a DNA mimic that binds to histone-like HU protein. Nucleic Acids Res. 41, 5127-5138.

(20) Hegde, S. S., Vetting, M. W., Roderick, S. L., Mitchenall, L. A., Maxwell, A., Takiff, H. E., and Blanchard, J. S. (2005) A fluoroquinolone resistance protein from Mycobacterium tuberculosis that mimics DNA. Science 308, 1480-1483.

(21) Bochkareva, E., Kaustov, L., Ayed, A., Yi, G. S., Lu, Y., PinedaLucena, A., Liao, J. C., Okorokov, A. L., Milner, J., Arrowsmith, C. H., and Bochkarev, A. (2005) Single-stranded DNA mimicry in the P53 transactivation domain interaction with replication protein A. Proc. Natl. Acad. Sci. U.S.A. 102, 15412-15417.

(22) Ramirez, B. E., Voloshin, O. N., Camerini-Otero, R. D., and Bax, A. (2000) Solution structure of DinI provides insight into its mode of RecA inactivation. Protein Sci. 9, 2161-2169.

(23) Court, R., Cook, N., Saikrishnan, K., and Wigley, D. (2007) The crystal structure of $\lambda$-Gam protein suggests a model for RecBCD inhibition. J. Mol. Biol. 371, 25-33.

(24) Lusetti, S. L., Voloshin, O. N., Inman, R. B., Camerini-Otero, R. D., and Cox, M. M. (2004) The DinI protein stabilizes RecA protein filaments. J. Biol. Chem. 279, 30037-30046.

(25) Atanasiu, C., Su, T. J., Sturrock, S. S., and Dryden, D. T. (2002) Interaction of the Ocr gene 0.3 protein of bacteriophage $\mathrm{T} 7$ with EcoKI restriction/modification enzyme. Nucleic Acids Res. 30, 39363944. 
(26) Roberts, G. A., Stephanou, A. S., Kanwar, N., Dawson, A., Cooper, L. P., Chen, K., Nutley, M., Cooper, A., Blakely, G. W., and Dryden, D. T. (2012) Exploring the DNA mimicry of the Ocr protein of phage T7. Nucleic Acids Res. 35, 5393-5401.

(27) Ghosh, M., Meiss, G., Pingoud, A. M., London, R. E., and Pedersen, L. C. (2007) The nuclease A-inhibitor complex is characterized by a novel metal ion bridge. J. Biol. Chem. 282, 56825690.

(28) De Backer, O., and Colson, C. (1991) Transfer of the genes for the StyLTI restriction-modification system of Salmonella typhimurium to strains lacking modification ability results in death of the recipient cells and degradation of their DNA. J. Bacteriol. 173, 1328-1330.

(29) Kennaway, C. K., Obarska-Kosinska, A., White, J. H., Tuszynska, I., Cooper, L. P., Bujnicki, J. M., Trinick, J., and Dryden, D. T. (2009) The structure of M.EcoKI Type I DNA methyltransferase with a DNA mimic antirestriction protein. Nucleic Acids Res. 37, 762-770.

(30) Wang, Z., and Mosbaugh, D. W. (1989) Uracil-DNA glycosylase inhibitor gene of bacteriophage PBS2 encodes a binding protein specific for uracil-DNA glycosylase. J. Biol. Chem. 264, 1163-1171.

(31) Enquist, L. W., and Skalka, A. (1973) Replication of bacteriophage $\lambda$ DNA dependent on the function of host and viral genes. I. Interaction of red, gam and rec. J. Mol. Biol. 75, 185-212.

(32) Murphy, K. C. (1991) $\lambda$ Gam protein inhibits the helicase and M-stimulated recombination activities of Escherichia coli RecBCD enzyme. J. Bacteriol. 173, 5808-5821.

(33) Wang, H. C., Wang, H. C., Leu, J. H., Kou, G. H., Wang, A. H. J., and Lo, C. F. (2007) Protein expression profiling of the shrimp cellular response to white spot syndrome virus infection. Dev. Comp. Immunol. 31, 672-686.

(34) Patat, S. A., Carnegie, R. B., Kingsbury, C., Gross, P. S., Chapman, R., and Schey, K. L. (2004) Antimicrobial activity of histones from hemocytes of the Pacific white shrimp. Eur. J. Biochem. 271, 4825-4833.

(35) Yasuda, T., Morimatsu, K., Horii, T., Nagata, T., and Ohmori, H. (1998) Inhibition of Escherichia coli RecA coprotease activities by DinI. EMBO J. 17, 3207-3216.

(36) Yasuda, T., Morimatsu, K., Kato, R., Usukura, J., Takahashi, M., and Ohmori, H. (2001) Physical interactions between DinI and RecA nucleoprotein filament for the regulation of SOS mutagenesis. EMBO J. 20, 1192-1202.

(37) Galkin, V. E., Britt, R. L., Bane, L. B., Yu, X., Cox, M. M., and Egelman, E. H. (2011) Two modes of binding of DinI to RecA filament provide a new insight into the regulation of SOS response by DinI protein. J. Mol. Biol. 408, 815-824.

(38) Chilley, P. M., and Wilkins, B. M. (1995) Distribution of the ardA family of antirestriction genes on conjugative plasmids. Microbiology 141, 2157-2164.

(39) Zavilgelsky, G. B., Kotova, V. Y., and Rastorguev, S. M. (2008) Comparative analysis of anti-restriction activities of ArdA (ColIb-P9) and Ocr (T7) proteins. Biochemistry (Moscow) 73, 906-911.

(40) Roberts, G. A., Chen, K., Bower, E. K., Madrzak, J., Woods, A., Barker, A. M., Cooper, L. P., White, J. H., Blakely, G. W., Manfield, I., and Dryden, D. T. (2013) Mutations of the domain forming the dimeric interface of the ArdA protein affect dimerization and antimodification activity but not antirestriction activity. FEBS J. 280, 4903-4914.

(41) Montero, C., Mateu, G., Rodriguez, R., and Takiff, H. (2001) Intrinsic resistance of Mycobacterium smegmatis to fluoroquinolones may be influenced by new pentapeptide protein MfpA. Antimicrob. Agents Chemother. 45, 3387-3392.

(42) Tran, J. H., and Jacoby, G. A. (2002) Mechanism of plasmidmediated quinolone resistance. Proc. Natl. Acad. Sci. U.S.A. 16, 56385642.

(43) Vetting, M. W., Hegde, S. S., and Blanchard, J. S. (2009) Crystallization of a pentapeptide-repeat protein by reductive cyclic pentylation of free amines with glutaraldehyde. Acta Crystallogr. D65, $462-469$.

(44) Vetting, M. W., Hegde, S. S., Zhang, Y., and Blanchard, J. S. (2011) Pentapeptide-repeat proteins that act as topoisomerase poison resistance factors have a common dimer interface. Acta Crystallogr. F67, 296-302.

(45) Vetting, M. W., Hegde, S. S., Wang, M., Jacoby, G. A., Hooper, D. C., and Blanchard, J. S. (2011) Structure of QnrB1, a plasmidmediated fluoroquinolone resistance factor. J. Biol. Chem. 286, 2526525273.

(46) Ghosh, M., Meiss, G., Pingoud, A., London, R. E., and Pedersen, L. C. (2005) Structural insights into the mechanism of nuclease A, a $\beta \beta \alpha$ metal nuclease from Anabaena. J. Biol. Chem. 280, 27990-27997.

(47) López-Rubio, J. J., Elías-Arnanz, M., Padmanabhan, S., and Murillo, F. J. (2002) A repressor-antirepressor pair links two loci controlling light-induced carotenogenesis in Myxococcus xanthus. J. Biol. Chem. 277, 7262-7270.

(48) Whitworth, D. E., and Hodgson, D. A. (2001) Light-induced carotenogenesis in Myxococcus xanthus: Evidence that CarS acts as an anti-repressor of CarA. Mol. Microbiol. 42, 809-819.

(49) López-Rubio, J. J., Padmanabhan, S., Lázaro, J. M., Salas, M., Murillo, F. J., and Elías-Arnanz, M. (2004) Operator design and mechanism for CarA repressor-mediated downregulation of the photoinducible carB operon in Myxococcus xanthus. J. Biol. Chem. 279, 28945-28953.

(50) Stabler, R. A., Marsden, G. L., Witney, A. A., Li, Y., Bentley, S. D., Tang, C. M., and Hinds, J. (2005) Identification of pathogenspecific genes through microarray analysis of pathogenic and commensal Neisseria species. Microbiology 151, 2907-2922.

(51) Exley, R. M., Sim, R., Goodwin, L., Winterbotham, M., Schneider, M. C., Read, R. C., and Tang, C. M. (2009) Identification of meningococcal genes necessary for colonization of human upper airway tissue. Infect. Immun. 77, 45-51.

(52) Sire, J., Quérat, G., Esnault, C., and Priet, S. (2008) Uracil within DNA: An actor of antiviral immunity. Retrovirology 5, 45.

(53) Chen, R., Wang, H., and Mansky, L. M. (2002) Roles of uracilDNA glycosylase and dUTPase in virus replication. J. Gen. Virol. 83, 2339-2345.

(54) Kruse, J. P., and Gu, W. (2009) Modes of P53 regulation. Cell 137, 609-622.

(55) Dutta, A., Ruppert, J. M., Aster, J. C., and Winchester, E. (1993) Inhibition of DNA replication factor RPA by P53. Nature 365, 79-82.

(56) Romanova, L. Y., Willers, H., Blagosklonny, M. V., and Powell, S. N. (2004) The interaction of P53 with replication protein A mediates suppression of homologous recombination. Oncogene 23, 9025-9033.

(57) Sommers, J. A., Sharma, S., Doherty, K. M., Karmakar, P., Yang, Q., Kenny, M. K., Harris, C. C., and Brosh, R. M., Jr. (2005) P53 modulates RPA-dependent and RPA-independent WRN helicase activity. Cancer Res. 65, 1223-1233.

(58) Rajagopalan, S., Andreeva, A., Teufel, D. P., Freund, S. M., and Fersht, A. R. (2009) Interaction between the transactivation domain of P53 and PC4 exemplifies acidic activation domains as single-stranded DNA mimics. J. Biol. Chem. 284, 21728-21737.

(59) Ge, H., and Roeder, R. G. (1994) Purification, cloning, and characterization of a human coactivator, $\mathrm{PC} 4$, that mediates transcriptional activation of class II genes. Cell 78, 513-523.

(60) Langlois, C., Mas, C., Di Lello, P., Jenkins, L. M., Legault, P., and Omichinski, J. G. (2008) NMR structure of the complex between the Tfb1 subunit of TFIIH and the activation domain of VP16: structural similarities between VP16 and P53. J. Am. Chem. Soc. 130, 10596-10604.

(61) Zhong, L., Wang, Y., Kannan, P., and Tainsky, M. A. (2003) Functional characterization of the interacting domains of the positive coactivator PC4 with the transcription factor AP-2 $\alpha$. Gene 320, 155164.

(62) Tora, L. (2002) A unified nomenclature for TATA box binding protein (TBP)-associated factors (TAFs) involved in RNA polymerase II transcription. Genes Dev. 16, 673-675.

(63) Studier, F. W. (1975) Gene 0.3 of bacteriophage T7 acts to overcome the DNA restriction system of the host. J. Mol. Biol. 15, 283-295. 
(64) Zhang, G., Wang, W., Deng, A., Sun, Z., Zhang, Y., Liang, Y., Che, Y., and Wen, T. (2012) A mimicking-of-DNA-methylationpatterns pipeline for overcoming the restriction barrier of bacteria. PLoS Genet. 8, e1002987.

(65) DelProposto, J., Majmudar, C. Y., Smith, J. L., and Brown, W. C. (2009) Mocr: A novel fusion tag for enhancing solubility that is compatible with structural biology applications. Protein Expression Purif. 63, 40-49.

(66) Ratner, D. (1974) The interaction bacterial and phage proteins with immobilized Escherichia coli RNA polymerase. J. Mol. Biol. 88, 373-383. 